\title{
Control of a VanderLugt correlator using a single 8-bit frame grabber
}

\author{
I. Labastida, A. Carnicer, E. Martín-Badosa, S. Vallmitjana, I. Juvells \\ Universitat de Barcelona, Departament de Física Aplicada i Òptica \\ Diagonal 647, E08028 Barcelona, Tel. + 34 934021202, Fax + 34 934021142, \\ e-mail: nasi@optica.ub.es
}

\begin{abstract}
We analyze in depth several engineering problems regarding the construction of a VanderLugt correlator. Two liquid crystal devices are used in the input and Fourier planes and the large distances involved are reduced with the help of telephoto systems. An original method to control both modulators with a single 8-bit frame grabber and a single videoprojector electronics is presented. Problems related to pixel-by-pixel addressing and the phase modulation in the panels are also discussed. All the solutions proposed in this paper have been implemented and experimental correlation results using the setup have been obtained.
\end{abstract}

KEY WORDS: Pattern recognition, optical correlation, spatial light modulators, liquid crystal devices. 


\section{Introduction}

Recently, the reduction of geometrical distances in optical correlators, in order to build miniaturized systems, has become a necessity for realistic applications. This need has resulted in several studies and publications carried out by several research groups $[1,2,3,4,5]$. In general, the scope of these papers only focuses in certain parts of the problem.

Nowadays, the presence of liquid crystal devices (LCD) as spatial light modulators (SLM) [6] has become the usual way to display images and control the setup in optical information processing systems [7, 8]. Although high frame rate modulators are available in the market today, a possible way to obtain LCD panels is by removing them from relatively low cost commercial videoprojectors $[9,10]$.

These devices are designed for imaging purposes so they present high resolution although operate at video frame rate. Despite several correlators having been designed to work at high frame rate [4], there are some applications that require higher resolution. For instance, adaptive systems based on the joint transform correlation are less critical in terms of video rate but require more pixels to display both scenes. High resolution modulators are also required in broad-area search problems [11].

As a result of using pixelated panels, the viability study of building an optical correlator in a reduced space should not only be limited to a design of optical systems. It must include an analysis of the behaviour of the display devices. Another issue that has not been raised yet is the use of the minimum material needed to control simultaneously two LCDs in a VanderLugt correlator, which involves a duplication in the driving electronics.

In this paper we analyze in depth several engineering problems regarding the construction of a VanderLugt correlator with LCDs at the input and Fourier planes. In order to reduce the length of the setup we have designed two telephoto systems. A method to control both SLMs with a single 8-bit frame grabber and a single videoprojector electronics is presented. As has been demonstrated, 16 grey levels for scene and filter display are enough for successful correlation [12]. Consequently, there is no need to use more sophisticated equipment. We have solved the problems related to pixel-by-pixel control when displaying the data from the frame grabber on the LCDs. An accurate analysis of the phase modulation capability of the panels is also discussed. Experimental results obtained with this setup are presented.

The paper is organized as follows: in Section 2 we describe and characterize the SLM devices used. In Section 3 we introduce a method to implement the pixel-by-pixel control from the frame grabber to the LCDs. It is based on a controlled zoom of the stored image in the frame grabber. In Section 4 we present an original procedure for simultaneously addressing both SLMs with a single low cost 8-bit frame grabber. The study of the correlator using telephoto systems to match the pixel structure of the SLMs and to reduce the distances involved is presented in Section 5 whereas in Section 6 several optical results are carried out. Finally, the conclusions can be found in Section 7 .

\section{Liquid crystal devices characterization}

The LCDs used in this work have been removed from an Epson VP-100PS videoprojector, shown in Figure 1. However, the electronics of the videoprojector still drives the images displayed on the LCDs. Kirsch et al. [9] 
studied the characteristics of a similar piece of equipment, an Epson Crystal Image Videoprojector E1020, but we have found substantial differences between these two models.

Each videoprojector has three LCDs, one for each RGB channel. In the correlator we use only two of them, one at the input plane and the other one at the Fourier plane. The active area of these LCDs is $25.6 \times 19.8 \mathrm{~mm}$, which corresponds to 320x264 pixels. The size of the pixels is $55 \times 50 \mu \mathrm{m}$ and the center-to-center distance is 80 $\mu \mathrm{m}$, horizontally, and $75 \mu \mathrm{m}$, vertically. Figures 1 and 2 show a picture of an LCD and a schematic drawing of its characteristics. As shown in the figure, a small fraction of the active area is not used by the electronics to display images (see details in Section 3.2).

The frame grabber used in the experiments is a Matrox PIP-1024B with an RGB output. This digitizer board stores an image of $512 \times 512$ pixels with 256 grey levels ( 8 bits per pixel).

\subsection{Operating curves of the LCDs}

The operating curves of the LCDs give information on the way in which these devices modulate light depending on the grey level assigned to each pixel $[13,14,15,16]$. The light can be modulated in amplitude, in phase or in both simultaneously [17]. This complex transmittance depends on the polarization state of light and on the addressing voltage applied to each pixel. When placing an LCD between two polarizers (polarizer and analyzer) different configurations of the device, that give rise to the different operating curves, depend on the position of the polarizers as well as on the potentiometer control positions (bright, contrast and color) of the videoprojector. When a single videoprojector is used to address two LCDs the aforementioned controls have to be set in a compromise position and the final operating curve adjustement has to be carried out by rotating the polarizers.

The choice of the operating curves for each LCD has to be taken into account in order to optimize the results in the correlator $[18,19]$. The most common configurations used to display images on the LCDs are:

High Contrast (HC): In this configuration the LCD contrast ratio should be as high as possible, although there may be a significant phase modulation.

Amplitude Mostly (AM): In this case the LCD has to modulate light only in amplitude and the phase variation should be zero. In fact there is always a small phase modulation [17]. With this configuration the contrast ratio is usually much lower than with the $\mathrm{HC}$ one.

Phase Mostly(PM): Unlike the AM configuration, with this operating curve the light should be modulated only in phase but actually there is always a small variation in amplitude.

The method used to characterize the LCDs, i.e. to find the operating curves, is divided into two parts. The amplitude modulation is obtained directly from the ratio between the input light and the output light for a range of grey levels. To measure the phase variation we have used an interferometric-based method. The LCD is placed in one of the arms of a Mach-Zehnder interferometer. The method consists of the measurement of the relative phase shift between several pairs of grey levels displayed on the panel: half of the screen always remains with a constant grey-level value, while different values are displayed on the other half. A wider explanation of the procedure can be found in Reference [15]. 
For the LCD used to display the scene we have found two operating curves corresponding to a HC and an AM configurations (Figure 3) while a PM configuration curve has been obtained for the second LCD (see Figure 4). These three curves have been found with a single position of the videoprojector potentiometer controls. The contrast ratio achieved with our HC curve is 100:1 while the associated phase modulation reaches a maximum of $0.9 \pi$ radians. On the other hand, with our AM configuration the contrast ratio is 10:1 and the maximum phase variation is only $0.12 \pi$ radians. Finally, our PM curve gives $1.88 \pi$ radians of phase modulation with a low amplitude variation (1:0.7).

\section{Addressing of the LCDs}

In order to deal simultaneously with two LCDs, usually two control systems are needed to manage the signal. In this paper we present an original method to control both SLMs with a single electronic system. The LCDs are driven by the electronics of a videoprojector and a single frame grabber is used to send the images, scene and filter, to the devices. With this method we reduce the equipment involved in the correlator because only one displaying system is required, obviously gaining in simplicity and economy.

In this section we also present a pixel-by-pixel addressing method in order to have a correct display of the images on the LCDs.

\subsection{Storage of two images in a single 8-bit frame grabber}

The frame grabber we use stores an image of $512 \times 512$ pixels of 8 bits. To deal with two images the 8 -bit word, or byte, has to be shared by the pixels of both images. In our method, the most significant nibble (4 bits) is occupied by the scene and the less significant nibble is used by the filter. Therefore, the number of grey levels of each image has to be reduced from 256 to 16 to be codified into a nibble. This is equivalent to stripping off the less significant nibble of the pixels of each image. Then the grey level stored in the frame grabber is generated by joining the two resulting nibbles into a single bite. Figure 5 summarizes the procedure. The reduction to 16 grey levels is not significant in terms of correlation performance as reported [12].

Since the digitizer has an RGB output it is possible to create different look-up-tables (LUT) for each channel. By using two appropriate LUTs, the two images information stored in a single byte can be separated in two different channels. Figures 7 and 8 show the LUTs designed for the green and the red channels, respectively. As it can be seen in the figures, only the most significant nibble of the new generated byte are relevant for the green channel while the output of the red channel only depends on the less significant nibble. The RGB output of the frame grabber is connected to the RGB input of the videoprojector. Note that the blue channel is not used.

The use of a single videoprojector presents an additional advantage with respect to driving the LCDs with two independent electronics, because in the latter case an accurate adjustment of the synchronism signal is needed.

The main advantage of this method is to reduce the equipment involved in the setup though there are a few disadvantages. The two images should be the same size and should be stored in the same memory position of 
the frame grabber. The potentiometer controls of the electronics have to be fixed in the same position for all the possible configurations of both LCDs. Another disadvantage is the reduction of the number of grey levels of the images, though it is not so important, as mentioned before. Finally, it has been seen that there is cross-talk between the two LCDs. Empirically it has been observed, by means of an oscilloscope, that for a certain position of the color control this phenomenon disappears almost completely.

\subsection{Managing pixel-by-pixel addressing from the frame grabber to the LCDs}

Although the nominal space bandwidth product of the devices is $320 x 264$ pixels, we have found that only $310 \times 242$ can be used in our setup to display the images. On the other hand, the bandwidth of the digitized image in the frame grabber is $512 \times 512$ pixels. We have observed that the first 28 rows and 34 columns of the stored image are lost when addressing the LCDs with the frame grabber. Therefore, the real bandwidth of the digitized image is reduced to $478 \times 484$ pixels.

The 484 rows are averaged two by two with an odd predominance, as it is shown in Figure 6, becoming the 242 active rows of the LCD. On the other hand, the 478 columns in the frame grabber become 310 interpolated columns. In practice, every third column is lost.

A procedure to maintain the pixel-by-pixel addressing of the images is presented. Other methods to achieve pixel-by-pixel control have been recently reported [20]. The images have to be sized to a maximum of 310x242 pixels. The rows are duplicated and a linear interpolated zoom is applied to the columns with a factor $1.542(=$ $\left.\frac{478}{310}\right)$. With this method the pixel-to-pixel control of images from the frame grabber to the LCDs is quite accurate, although the column addressing is more critical than the row one.

\section{Telephoto converging VanderLugt correlator}

The converging VanderLugt correlator is a modification of the classical $4 \mathrm{f}$ architecture [21]. The main problem that presents the $4 \mathrm{f}$ correlator is the need of large distances to work in the optical bench with the suitable elements and the appropriate scale. The optical set up of the converging correlator is sketched in Figure 9, [22].

This correlator is based on the coupling of two diffractometers with converging lenses. With this architecture the required distances are shorter than for the $4 \mathrm{f}$ system. It is still possible to further reduce the distances using diverging lenses as it is explained in the present section. This architecture has another advantage in that it allows the modification of the scale of the Fourier transforms without changing neither the lenses nor the distances between them. This versatility simplifies the fitting between the optical transform and the filter. The matching has to be done accurately because the correlation results are extremely sensitive to misaligments [23].

According to Figure 9 it is possible to describe how the converging correlator works. The system is illuminated with a monochromatic light of wavelength $\lambda$ expanded from point source O. The scene, denoted by the twodimensional function $g(x, y)$, is placed after the first converging lens at an arbitrary distance. The Fourier transform of the scene, $G(u, v)$, multiplied by a quadratic phase, appears centered at the point O', image point of $\mathrm{O}$ through the first lens. In this position, or Fourier plane, it is where the filter, $F^{*}(u, v)$, is placed and where the product of the two functions, $G(u, v) F^{*}(u, v)$, is optically obtained. The second lens forms the image of the 
scene at a plane passing through P', image point of point P. The Fourier transform of the product appears at this plane, thus producing the correlation between the two functions $g(x, y)$ and $f(x, y)$. This plane is called the correlation plane.

In order to study the scales involved in the correlator it is possible to do a mathematical analysis of the system $[24,25]$. In a first order approximation, a spherical lens with a focal length f' can be thought of as a a system which introduces a phase factor $\psi\left(x, y ;-F^{\prime}\right)=e^{-i \frac{\pi}{\lambda f^{\prime}}\left(x^{2}+y^{2}\right)}$. If the scene is denoted by $g(x, y)$, the amplitude distribution in the Fourier plane is:

$$
\mathcal{A}\left(x^{\prime}, y^{\prime}\right)=Q \int \psi\left(-\alpha,-\beta ; L_{1}\right) \psi^{*}\left(\alpha, \beta ; F_{1}^{\prime}\right) \psi\left(\alpha-x, \beta-y ; L_{2}\right) g_{(x, y)} \psi_{\left(x-x^{\prime}, y-y^{\prime} ; L_{3}\right)} d \alpha d \beta d x d y
$$

where $Q$ is a constant and $(\alpha, \beta)$ are the coordinates of the plane where the first lens is placed. Integrating with respect to $\alpha$ and $\beta$ and, after a little of algebra, equation 1 reduces to:

$$
\mathcal{A}\left(x^{\prime}, y^{\prime}\right)=Q \psi_{\left(x^{\prime}, y^{\prime} ; L_{3}\right)} \int \psi_{\left(x, y ; L_{2}+L_{3}-\frac{L_{2}^{2}}{L_{1}-F_{1}^{\prime}+L_{2}}\right)} g(x, y) e^{-i \frac{2 \pi L_{3}}{\lambda}\left(x x^{\prime}+y y^{\prime}\right)} d x d y
$$

If $\psi\left(x, y ; L_{2}+L_{3}-\frac{L_{2}^{2}}{L_{1}-F_{1}^{\prime}+L_{2}}\right)=1$, then $\mathcal{A}\left(x^{\prime}, y^{\prime}\right)$ will be the Fourier transform of $g(x, y)$, except for the unimportant constant factor $Q$. This condition is fullfilled when the plane ( $\left.\mathrm{x}^{\prime}, \mathrm{y}^{\prime}\right)$ is placed at the position $\mathrm{O}^{\prime}$, image point of $\mathrm{O}$ :

$$
\psi\left(x, y ; L_{2}+L_{3}-\frac{L_{2}^{2}}{L_{1}-F_{1}^{\prime}+L_{2}}\right)=1 \quad \rightarrow \quad \frac{1}{f_{1}^{\prime}}=\frac{1}{l_{1}}+\frac{1}{l_{2}+l_{3}}
$$

then:

$$
\mathcal{A}\left(x^{\prime}, y^{\prime}\right)=Q \psi_{\left(x^{\prime}, y^{\prime}, L_{3}\right)} G(u, v) \quad \text { where } \quad u=\frac{L_{3} x^{\prime}}{\lambda} \quad \text { and } \quad v=\frac{L_{3} y^{\prime}}{\lambda}
$$

In an analogous way, we derive the amplitude distribution in the correlation plane $(\mathrm{x} ", \mathrm{y} ")$ :

$$
\begin{array}{r}
\mathcal{C}\left(x^{\prime \prime}, y^{\prime \prime}\right)=L_{4} L_{5} \int \mathcal{A}\left(x^{\prime}, y^{\prime}\right) F^{*}{ }_{\left(x^{\prime}, y^{\prime}\right) \psi\left(x^{\prime}-\alpha^{\prime}, y^{\prime}-\beta^{\prime} ; L_{4}\right)} \psi^{*}\left(\alpha^{\prime}, \beta^{\prime} ; F_{2}^{\prime}\right) \\
\psi\left(\alpha^{\prime}-x^{\prime \prime}, \beta^{\prime}-y^{\prime \prime} ; L_{2}\right) d \alpha^{\prime} d \beta^{\prime} d x^{\prime} d y^{\prime}
\end{array}
$$

where $F^{*}\left(x^{\prime}, y^{\prime}\right)$ represents the filter. In order to reduce the latter expression to a Fourier transform the following condition has to be met:

$$
\frac{1}{f_{2}^{\prime}}=\frac{1}{l_{5}}+\frac{1}{l_{3}+l_{4}}
$$

i.e. the correlation plane is found at the image plane of the scene through the second lens, $F l_{2}$. Finally, the amplitude distribution is:

$$
\begin{array}{r}
\mathcal{C}\left(x^{\prime \prime}, y^{\prime \prime}\right)=Q \psi\left(x^{\prime \prime}, y^{\prime \prime} ; l_{5}-\frac{L_{5}^{2}}{L_{4}+L_{5}-F_{2}^{\prime}}\right) \int G\left(L_{3} x^{\prime}, L_{3} y^{\prime}\right) F^{*}\left(x^{\prime}, y^{\prime}\right) \\
e^{-i \frac{2 \pi}{\lambda} \frac{-L_{4} L_{5}}{L_{4}+L_{5}-F_{2}^{\prime}}\left(x^{\prime} x^{\prime \prime}+y^{\prime} y^{\prime \prime}\right)} d x^{\prime} d y^{\prime}
\end{array}
$$

which is, aside of non-detectable phase factors, the Fourier transform of the product of the two functions. This transform produces the correlation between the scene, $g(x, y)$, and the image reference, $f(x, y)$.

\subsection{Distance matching}

To design the correlator, several considerations concerning the scales and distances involved in the optical setup should be taken into account. We define the diffraction constant $(K)$ as the scale factor between the Fourier 
plane and the input plane. It can be calculated as $K=\lambda d$, where $\mathrm{d}$ is the distance between these two planes. In the converging correlator, the diffraction constant $K=\lambda l_{3}$ corresponding to lens $F l_{1}$ should be large enough to separate the diffraction orders generated by the pixelated structure of the first LCD. In general, the distance between orders has to be at least the size of the display [1]. Nevertheless, the required separation can be reduced to the size of the image, in this case $256 \times 256$ pixels. As we have pointed out in Section 2 the distance between pixels in the LCD is $80 \mu m$ horizontally and $75 \mu m$ vertically. Using an input LCD with a non squared pixel grid produces a distorsion in the optical Fourier transform. Thus, the filter image has to be modified for a correct match. The simplest way to have a good fitting between filter and Fourier transform is to rotate $90^{\circ}$ the second LCD.

Therefore the minimum diffraction constant needed is $K=256 \times 0.08 \times 0.075=1.54 \mathrm{~mm}^{2}$, where the factor $256 \times 0.08 \mathrm{~mm}$ is the size of the filter image on the second LCD and $0.075 \mathrm{~mm}$ is the pixel separation in the input LCD.

From the minimum value of $K$ required, the distance between the input and the Fourier planes has to be at least $l_{3}=2.436 \mathrm{~m}$, with a He-Ne laser $(\lambda=633 \mathrm{~nm})$ as illumination source. Using a classical $4 \mathrm{f}$ correlator, this distance would be $2 l_{3}=4.87 \mathrm{~m}$.

Although the total length of the setup is reduced with the converging correlator, is advisable the use of telephoto systems instead of single lenses. By using couples of converging and diverging lenses, tunable high value diffraction constants can be achieved in a minimum space $[25,26]$. Using a single converging lens, the diffraction constant is $K=\lambda d$ (Figure 10). Rewriting $K$ in terms of the variables present in a telediffractometer (Figure 11),

$$
K_{\text {conv }}=\lambda(s-t)
$$

where $\mathrm{s}$ is the distance between the input plane and the diverging lens and $\mathrm{t}$ is the distance between the diverging lens and the image plane of the point source through the converging lens.

To determine the constant $K$ in a telediffractometer, it is possible to use the aforementioned expression $(K=\lambda d)$ where $\mathrm{d}$ is now the distance between the image of the input plane through the diverging lens and the Fourier plane $\left(d=-s^{\prime}+r\right)$. The magnification $\beta=\frac{-s^{\prime}}{s}$ of the input plane through the lens should be considered as follows:

$$
K_{\text {conv }+ \text { div }}=\lambda\left(-s^{\prime}+r\right)\left[\frac{s}{-s^{\prime}}\right]
$$

Rewriting equations 8 and 9 in terms of the focal lengths it is possible to establish a comparison between both constants,

$$
\begin{aligned}
K_{\text {conv }} & =\lambda\left(s+\frac{r f_{d i v}^{\prime}}{f_{d i v}^{\prime}+r}\right) \\
K_{c o n v+d i v} & =\lambda\left(s+\frac{r f_{d i v}^{\prime}}{f_{d i v}^{\prime}}+\frac{r s}{f_{d i v}^{\prime}}\right)
\end{aligned}
$$

where $f_{d i v}^{\prime}$ is the absolute value of the focal length of the diverging lens. Analyzing these equations we can conclude that $K_{\text {conv+div }}>K_{c o n v}$. 


\section{Experimental results}

In this section we demonstrate the use of the correlator. A VanderLugt optical setup with two SLMs in the input and Fourier plane controlled by means of a single 8-bit frame grabber and telephoto systems to perform diffraction has been designed and built.

Figure 12 shows the three satellite scene used in the experiments. The target to detect is the smallest satellite, which is partially superimposed to the Earth. The original scene is $256 \times 256$ pixels and 8 bits per pixel (256 grey levels). This image is reduced to a nibble (4 bits) per pixel and a zoom is applied to ensure pixel-by-pixel control as explained in Section 3.2. This modified scene is displayed on the first LCD working with a HC operating curve.

A phase-only filter (POF) of the target (the smallest satellite) has been used, taking into account the phase introduced in the scene by the high contrast configuration of the first LCD. The POF is computed from the complex values of the scene displayed on te LCD: let $\mathrm{f}(\mathrm{x}, \mathrm{y})$ be the target and let $\hat{f}(x, y)$ the complex valued function obtained after modifying $f(x, y)$ with the operating curve of the LCD. Then, the POF is obtained in the usual way,

$$
\operatorname{POF}(u, v)=\frac{[\mathcal{F} \mathcal{T}(\hat{f}(x, y))]^{*}}{|\mathcal{F} \mathcal{T}(\hat{f}(x, y))|}
$$

Figure 13 shows the optical correlation between the scene and the POF matched to the smallest satellite. This result has been obtained with the second panel operating in the PM configuration.

A second experiment has been carried out. Now, the first LCD is turned to the AM configuration. Figure 14 shows the detection obtained in this case. Although the correlation is less noisy than in the first case, a smaller ammount of light in the correlation plane is observed.

\section{Conclusions}

In this paper we have demonstrated the possibility of simultaneously controlling two LCDs with a single 8-bit frame grabber and a single videoprojector electronics. The main advantage of the method proposed is the management of the correlator with the minimum equipment. Moreover, a complete study of the correlator involving i) the use of telephoto systems to reduce the distances involved, ii) a pseudo pixel-by-pixel images addressing from the frame grabber to the LCDs and iii) the analysis of the operating curves of the LCDs and their use in the design of the filters. The experimental optical results obtained show the good performance of the setup proposed.

\section{Acknowledgments}

This paper has been supported by CICYT (Comisión Interministerial de Ciencia y Tecnología) under the projects TAP94-0303 and TAP97-0454. I. Labastida acknowledges the support of Fundaci Bosch i Gimpera, project FBG-2216. 


\section{References}

[1] B. D. Bock, T. A. Crow, and M. K. Giles, "Design considerations for miniature optical correlation systems that use pixelated input and filter transducers," Proc. SPIE, vol. 1347, no. San Diego, pp. 297-309, 1990.

[2] C. M. Crandall, M. K. Giles, and N. Clark, "Performance limitations of miniature optical correlators," Proc. SPIE, vol. 1564, no. Orlando, pp. 98-109, 1991.

[3] J. A. Davis, M. A. Waring, G. W. Bach, R. A. Lilly, and D. M. Cottrell, "Compact optical correlator design," Appl. Opt., vol. 28, pp. 10-11, 1989.

[4] J. P. Karins, S. A. Mills, N. J. Szegedi, J. R. Ryan, L. G. Kelly, D. H. Goldstein, E. P. Augustus, and R. J. Wrangler, "Miniature ruggedized optical correlator for flight testing," Proc. SPIE, vol. 2237, pp. 48-53, 1994.

[5] H. Rajbenbach, S. Bann, P. Rfrgier, P. Joffre, J. P. Huignard, H. S. Buchkremer, A. S. Jensen, E. Rasmussen, K. H. Brenner, and G. Lohman, "Compact photorefractive correlator for robotic applications," Appl. Opt., vol. 31, pp. 5666-5674, 1992.

[6] U. Efron, "Spatial light modulators and applications for optical information processing," Proc. SPIE, vol. 960, pp. 180-203, 1988.

[7] D. A. Gregory, "Real-time pattern recognition using a modified liquid crystal television in a coherent optical correlator," Appl. Opt., vol. 25, pp. 467-469, 1986.

[8] N. Clark, C. M. Crandall, and M. K. Giles, "Using liquid crystal TVs in Vander Lugt optical correlators," Proc. SPIE, vol. 1564, no. Orlando, pp. 439-451, 1991.

[9] J. C. Kirsch, D. A. Gregory, M. W. Thie, and B. K. Jones, "Modulation characteristics of the Epson liquid crystal television," Opt. Eng., vol. 31, pp. 963-970, 1992.

[10] J. N. Duffey, B. K. Jones, J. A. Loudin, and J. Booth, "Optical characterization of the InFocus TVT-600 LCTV using custom device electronics," Proc. SPIE, vol. 2490, pp. 432-440, 1995.

[11] B. K. Jones and J. C. Kirsch, "Optical correlator comparison of near and far field ISAR imagery," Proc. SPIE, vol. 2490, pp. 312-317, 1995.

[12] Y. Sheng and G. Paul-Hus, "Optical on-axis imperfect phase-only correlator using liquid-crystal television," Appl. Opt., vol. 32, pp. 5782-5785, 1993.

[13] C. Soutar, S. E. Monroe, and J. Knopp, "Measurement of the complex transmittance of the Epson liquid crystal television," Opt. Eng., vol. 33, pp. 1061-1068, 1994.

[14] L. Gonçalves, D. Roberge, and Y. Sheng, "Programmable optical phase-mostly holograms with coupledmode modulation liquid-crystal television," Appl. Opt., vol. 34, pp. 1944-1950, 1995.

[15] E. Martín-Badosa, A. Carnicer, I. Juvells, and S. Vallmitjana, "Liquid crystal devices complex modulation characterization by interferometric data correlation," Meas. Sci. Technol, vol. 8, pp. 764-772, 1997.

[16] J. L. McClain, P. S. Erbach, D. A. Gregory, and F. T. S. Yu, "Spatial light modulators phase depth determination from optical diffraction information," Opt. Eng., vol. 35, pp. 951-954, 1996. 
[17] K. Lu and B. E. A. Saleh, "Theory and design of the liquid crystal TV as an optical spatial phase modulator," Opt. Eng., vol. 29, pp. 240-246, 1990.

[18] C. Soutar and S. E. Monroe, "Selection of operating curves of twisted-nematic liquid crystal televisions," Proc. SPIE, vol. 2240, no. Orlando, pp. 280-291, 1994.

[19] A. Skorucak and C. Soutar, "Selection criteria for spatial light modulator operating curves," Proc. SPIE, vol. 2752, no. Orlando, pp. 120-131, 1996.

[20] J. Knopp, M. V. Morelli, S. E. Monroe, and R. D. Juday, "Pixel-level control of an Epson LCTV SLM," Proc. SPIE, vol. 2752, no. Orlando, pp. 144-152, 1996.

[21] A. B. VanderLugt, "Signal detection by complex filtering," IEEE Transactions on Information Theory, vol. IT-10, pp. 139-145, 1964.

[22] D. Flannery, A. M. Biernacki, J. S. Loomis, and S. L. Cartwright, "Real-time coherent correlator using binary magnetooptic spatial light modulators at input and Fourier planes," Appl. Opt., vol. 25, p. 466, 1986.

[23] M. Montes-Usategui, S. E. Monroe, and R. D. Juday, "Automated self-alignement procedure for optical correlators," Opt. Eng., vol. 36, pp. 1782-1791, 1997.

[24] A. B. VanderLugt, "Operational notation for the analyisis of optical data-processing systems," Proc. IEEE, vol. 54, pp. 1055-1062, 1966.

[25] I. Juvells, S. Vallmitjana, and S. Bosch, "Analysis of a scale-tunable telephoto lens diffractometer and its use in optical correlation," J. Modern Optics, vol. 39, pp. 1107-1115, 1992.

[26] S. Bosch, S. Vallmitjana, I. Juvells, and J. F. Moneo, "Scale-tunable diffractometer for spatial light modulators. A design procedure," Proc. SPIE, vol. 1780, pp. 592-601, 1992. 


\section{List of Figures}

1 Epson VP-100PS videoprojector, inside view of the videoprojector and one of the LCDs removed

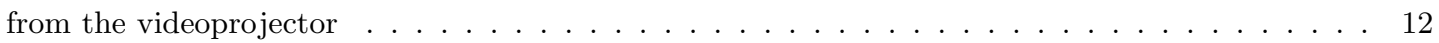

2 Schematic drawing of the LCD structure . . . . . . . . . . . . . . . . . . . 12

3 Operating curves of the LCD to display the scene . . . . . . . . . . . . . . . . . . . . 12

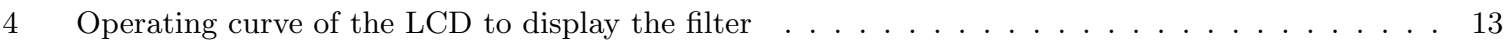

5 Method to control two images in the frame grabber . . . . . . . . . . . . . . 13

6 Image conversion from the frame grabber to the LCD $\ldots \ldots \ldots \ldots \ldots$

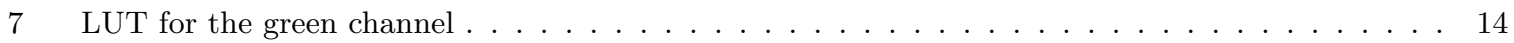

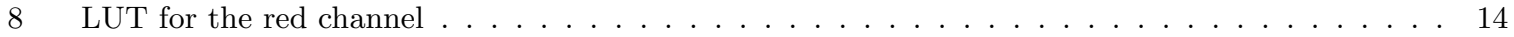

$9 \quad$ Converging VanderLugt correlator . . . . . . . . . . . . . . . . . . . . . . . . . 14

10 Diffractometer with a single converging lens . . . . . . . . . . . . . . . . 15

11 Telephoto system or telediffractometer . . . . . . . . . . . . . . . . . 15

12 Satellites and Earth scene . . . . . . . . . . . . . . . . . . . . . . . . 15

13 Experimental correlation. The first LCD is in the HC configuration . . . . . . . . . . . . 16

14 Experimental correlation. The first LCD is in the AM configuration . . . . . . . . . . . 16 

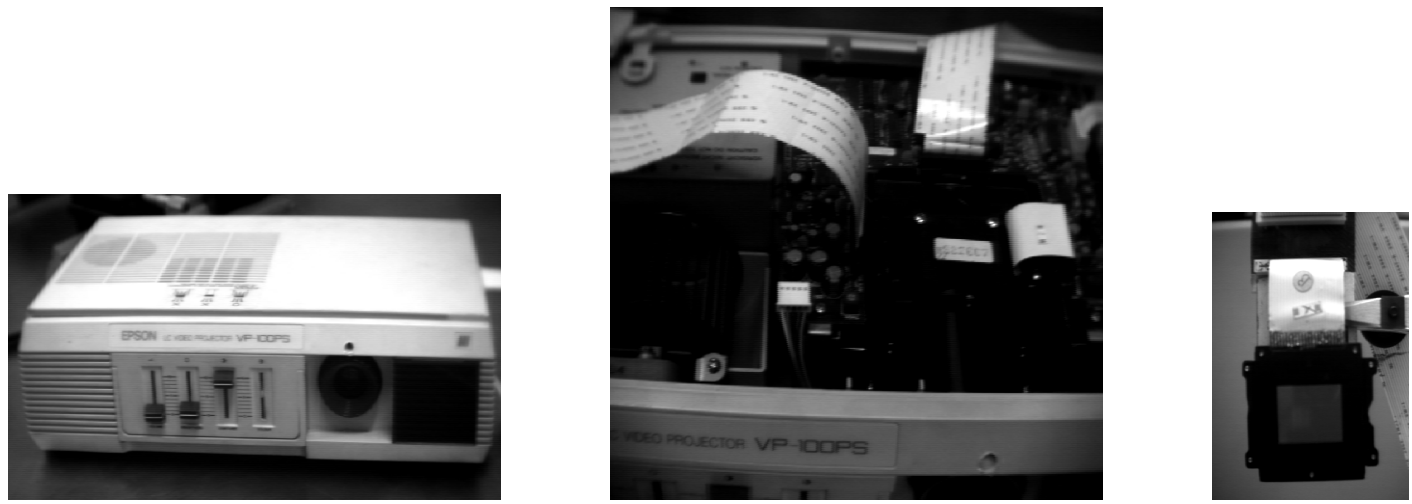

Figure 1: Epson VP-100PS videoprojector, inside view of the videoprojector and one of the LCDs removed from the videoprojector

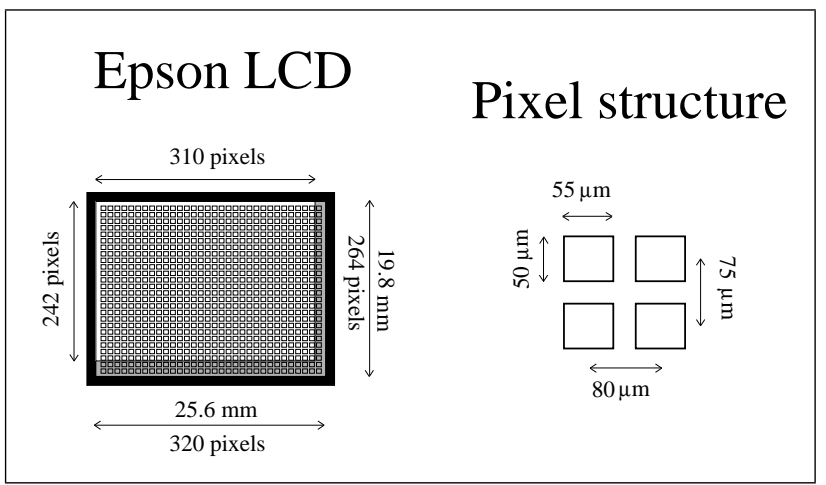

Figure 2: Schematic drawing of the LCD structure
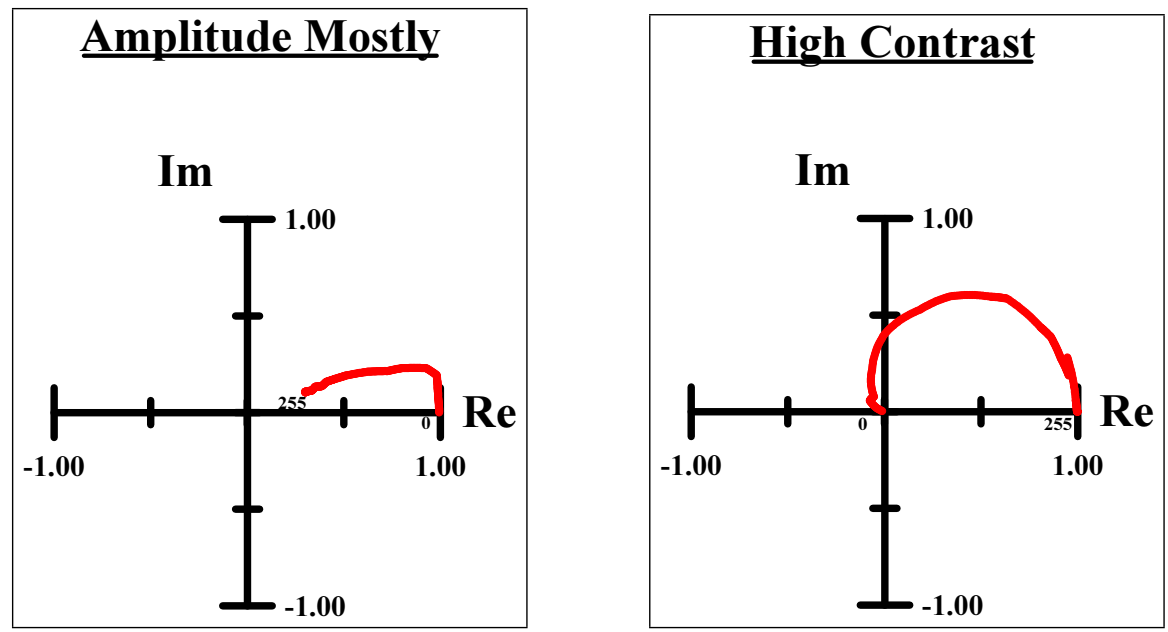

Figure 3: Operating curves of the LCD to display the scene 


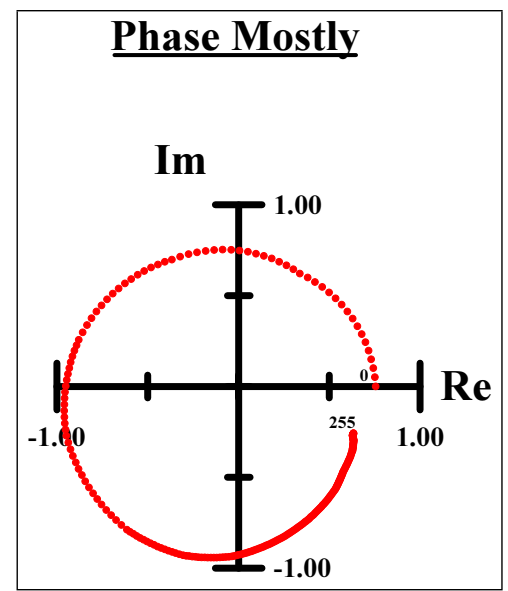

Figure 4: Operating curve of the LCD to display the filter

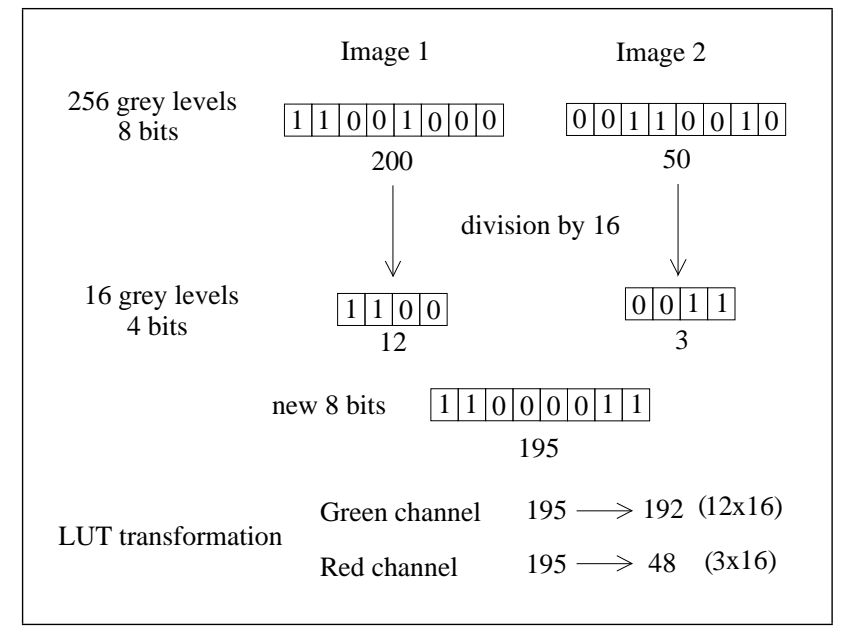

Figure 5: Method to control two images in the frame grabber

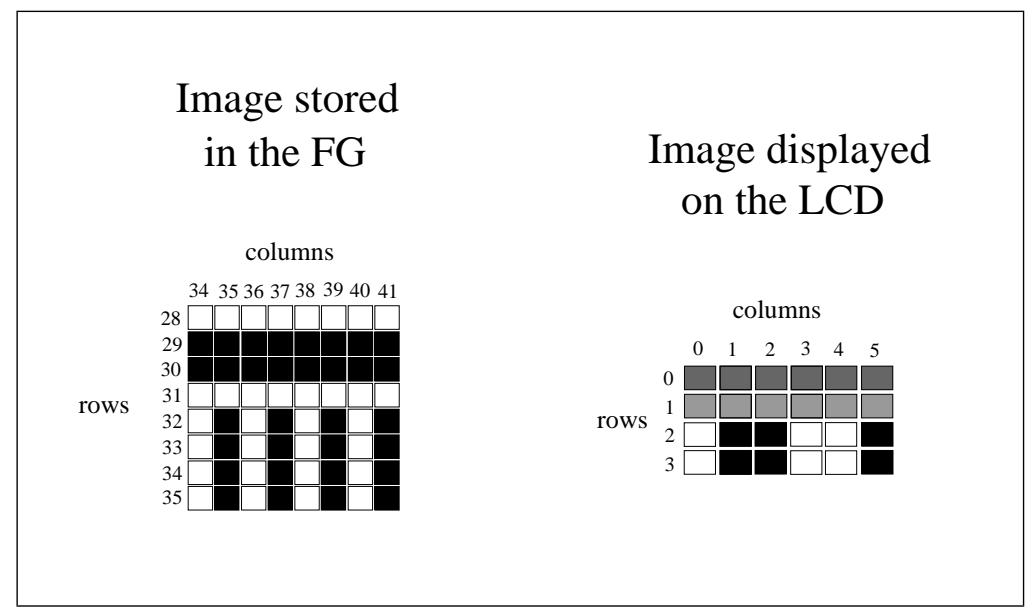

Figure 6: Image conversion from the frame grabber to the LCD 


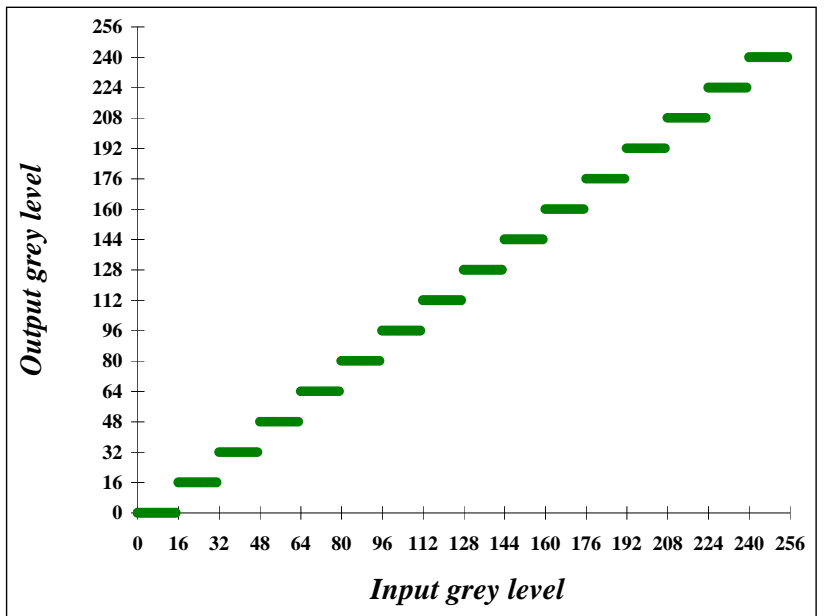

Figure 7: LUT for the green channel

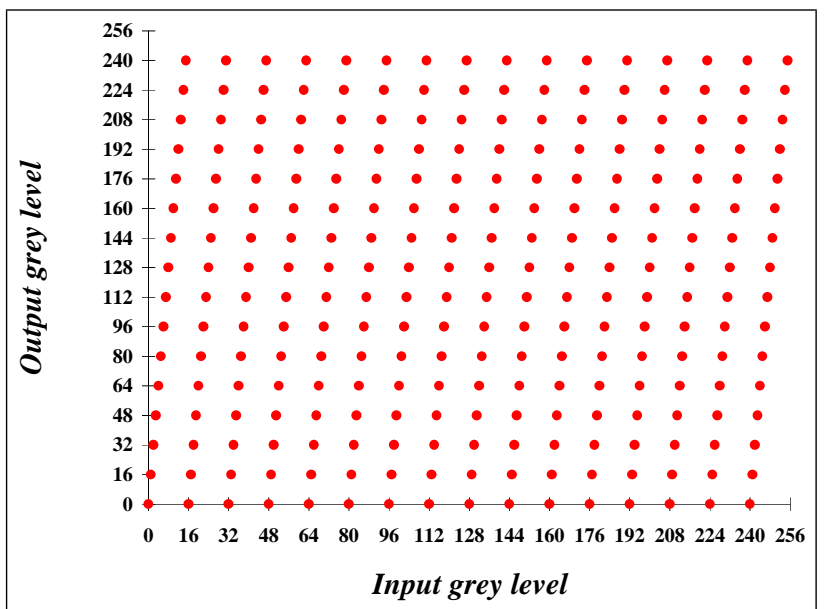

Figure 8: LUT for the red channel

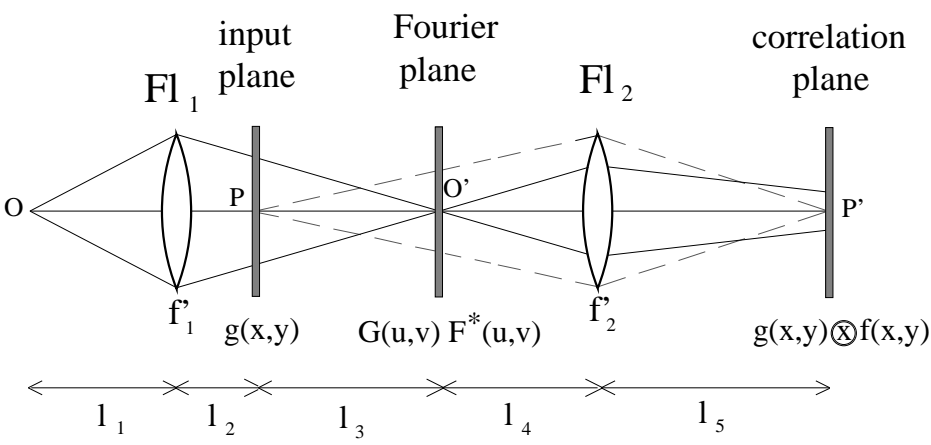

Figure 9: Converging VanderLugt correlator 


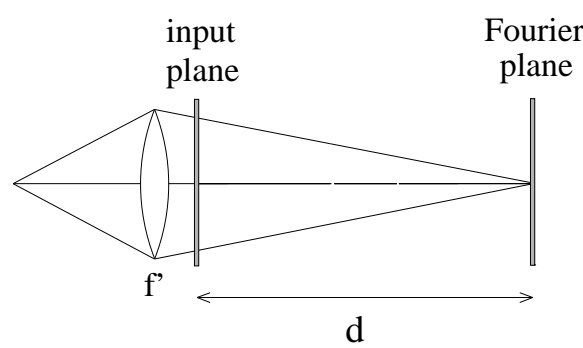

Figure 10: Diffractometer with a single converging lens

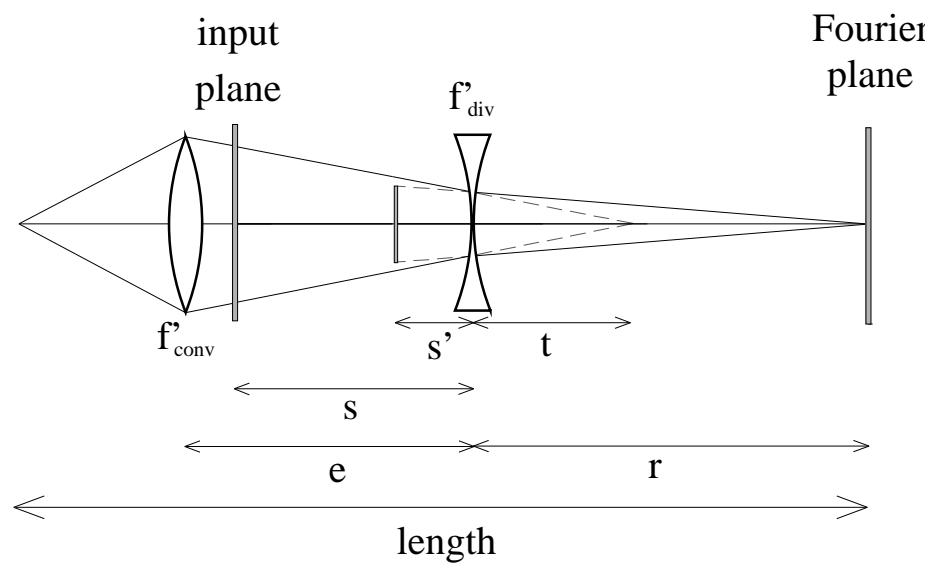

Figure 11: Telephoto system or telediffractometer

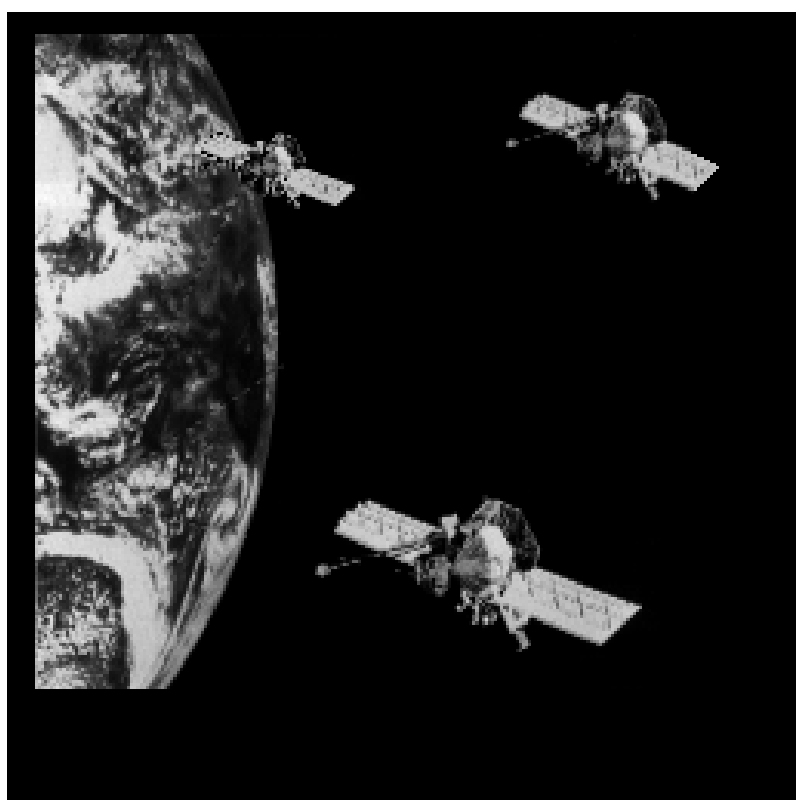

Figure 12: Satellites and Earth scene 


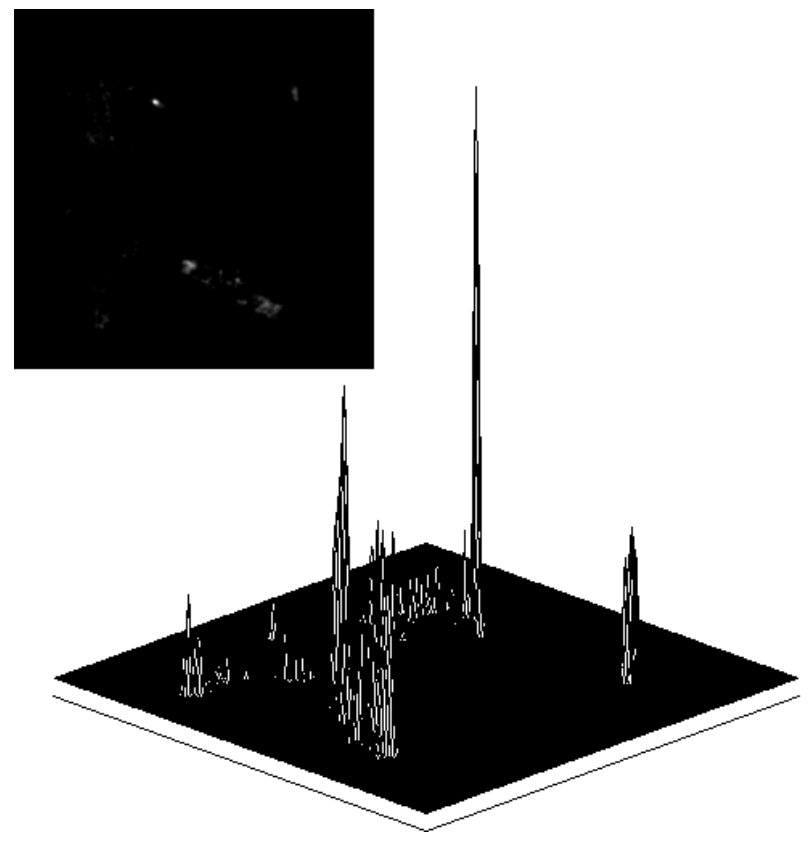

Figure 13: Experimental correlation. The first LCD is in the HC configuration

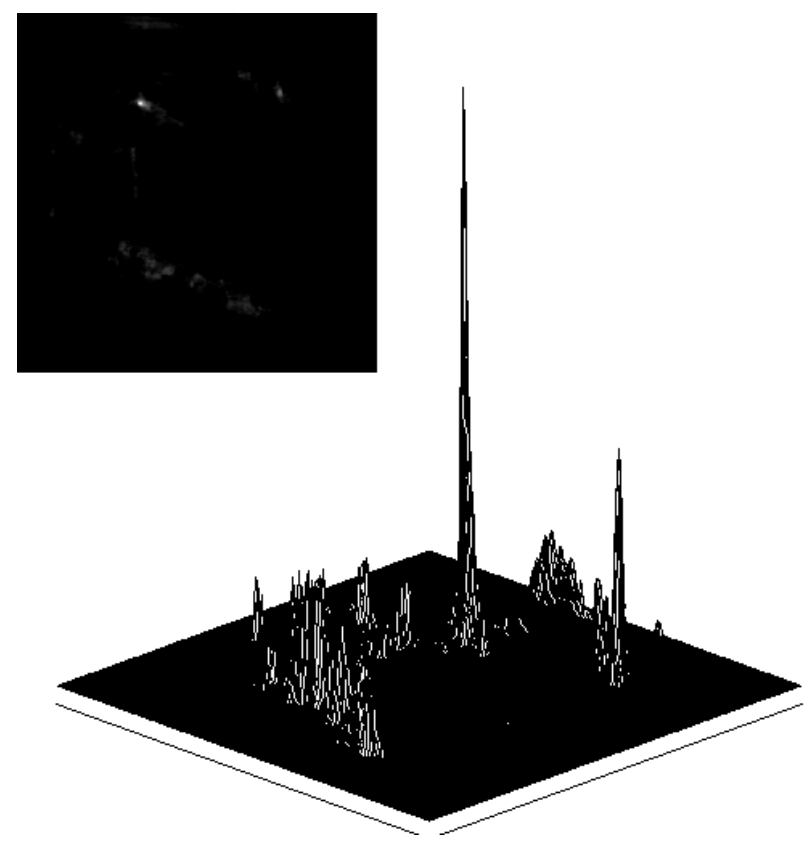

Figure 14: Experimental correlation. The first LCD is in the AM configuration 